The Astrophysical Journal, 685:1046-1051, 2008 October 1

(C) 2008. The American Astronomical Society. All rights reserved. Printed in U.S.A.

\title{
ON DUST EXTINCTION OF GAMMA-RAY BURST HOST GALAXIES
}

\author{
Aigen Li, ${ }^{1,2}$ S. L. Liang, ${ }^{1}$ D. A. KanN, ${ }^{3}$ D. M. Wei, ${ }^{2}$ S. Klose, ${ }^{3}$ and Y. J. WanG ${ }^{4}$ \\ Received 2008 February 25; accepted 2008 June 11
}

\begin{abstract}
Although it is well recognized that gamma-ray burst (GRB) afterglows are obscured and reddened by dust in their host galaxies, the wavelength dependence and quantity of dust extinction are still poorly known. Current studies on this mostly rely on fitting the afterglow spectral energy distributions (SEDs) with template extinction models. The inferred extinction (both quantity and wavelength dependence) and dust-to-gas ratios are often in disagreement with that obtained from dust depletion and X-ray spectroscopy studies. We argue that this discrepancy could result from the prior assumption of a template extinction law. We propose an analytical formula to approximate the GRB host extinction law. With the template extinction laws self-contained, and the capability of revealing extinction laws differing from the conventional ones, it is shown that this is a powerful approach in modeling the afterglow SEDs to derive GRB host extinction.
\end{abstract}

Subject headings: dust, extinction — gamma rays: bursts

\section{INTRODUCTION}

In addition to the Galactic foreground extinction, gamma-ray bursts (GRBs) and their afterglows are subject to extinction caused by the dust within their host galaxies. Evidence for this include the following:

1. Dark bursts. - An appreciable fraction of GRBs with X-ray and/or radio afterglows lack an optical afterglow (Jakobsson et al. 2004)..$^{5}$ A natural explanation for dark bursts is that they lie behind significant obscuring dust columns in their host galaxies, which effectively suppresses the optical light (although some dark bursts may be intrinsically faint or occur at high redshifts (say, $z \gtrsim 5$ ), where the Ly $\alpha$ break has moved through the optical bands, leading to absorption of the optical light by the Ly $\alpha$ forest). Indeed, Schady et al. (2007) found that the X-ray afterglows of GRBs not detected by UVOT were more affected by extinction than those of GRBs with detected UVOT counterparts. The recent detection of the near-infrared (IR) afterglows of some GRBs (which would have been considered as "dark bursts" since their afterglows were not detected in any bluer bands) provides another piece of evidence for dust obscuration (e.g., see Jaunsen et al. 2008; Tanvir et al. 2008).

2. Reddening.-Some GRB afterglows with low redshifts appear very red, due to effects of extinction - ultraviolet (UV)/ visible light is extinguished more by dust than red light (e.g., see Klose et al. 2000; Levan et al. 2006). Dust reddening is also indicated by the significant deviation of the optical/near-IR spectral energy distributions (SEDs) of many afterglows from that expected from standard models. Also, because of dust reddening, the Balmer line ratios in the spectra of some GRB host galaxies (e.g., see Djorgovski et al. 1998), known as the "Balmer decrement," deviate from the expected ratios for the standard case B

\footnotetext{
1 Department of Physics and Astronomy, University of Missouri, Columbia, MO 65211; lia@missouri.edu.

2 Purple Mountain Observatory, Chinese Academy of Sciences, Nanjing 210008, China.

3 Thüringer Landessternwarte Tautenburg, D-07778 Tautenburg, Germany.

4 Department of Physics, Hunan Normal University, Changsha 410071, China.

5 Prior to the launch of Swift, nearly $\sim 60 \%$ of the X-ray afterglows reportedly had no optical counterparts. Despite rapid and deep searches in the Swift era, it was found that $\sim \frac{1}{3}$ GRBs with bright X-ray afterglows remain undetected at optical wavelengths (Fiore et al. 2007; Schady et al. 2007).
}

recombination, which are fairly independent of physical conditions (Osterbrock \& Ferland 2006).

3. Depletion.-Dust-forming heavy elements such as Si and Fe were found to be substantially depleted from the gas phase in some host galaxies (e.g., see Savaglio et al. 2003). This indirectly shows the presence of dust in GRB host galaxies since the missing heavy elements must have been locked up in dust grains.

4. Connection between long GRBs and massive stars.-There are multiple strong lines of evidence that long-duration $(\gtrsim 2 s)$ GRBs are associated with the death of massive stars, occurring in regions of active star formation embedded in dense clouds of dust and gas (see Woosley \& Bloom 2006).

A precise knowledge of the extinction (quantity, wavelength dependence) and the nature (size, composition, and quantity) of the dust in GRB host galaxies is crucial for (1) correcting for the extinction of afterglows from X-ray to near-IR wavelengths to derive their intrinsic luminosities - this is particularly important for studying the luminosity distribution of GRB afterglows and their intrinsic SEDs (e.g., see Kann et al. 2008); (2) constraining the nature of the GRB progenitors (i.e., collapsing massive stars or merging neutron stars) - if long-duration GRBs are indeed linked to the collapse of massive stars, it is most likely that their optical and near-IR afterglows will suffer from significant attenuation in the star-forming molecular clouds heavily enshrouded by dust - the birth place of these short-lived $\left(\sim 10^{6} \mathrm{yr}\right)$ massive stars; (3) tracing the physical conditions of (and processes occurring in) the environments where GRBs occur which hold clues for understanding the mechanism for making a burst; e.g., a flat or gray extinction law for GRB host galaxies would imply a dense circumburst environment where dust undergoes coagulational growth or a preferential destruction of small grains; and (4) probing the interstellar medium (ISM) of high-redshift galaxies and the cosmic star formation history-because of their intense luminosity which allows their detection at cosmological distances, GRBs are a powerful tool to study the star formation history up to very high redshifts; e.g., the dust and extinction properties of GRB hosts would help us understand the nature of dark bursts and the dark burst fraction, which would place important constraints on the fraction of obscured star formation in the universe (e.g., see Djorgovski et al. 2001; Ramirez-Ruiz et al. 2002).

However, our current understanding of the dust extinction in GRB host galaxies is still very poor. Existing studies on this often 
draw conclusions in conflict with each other (see $\S 2$ for details). We argue that this could be caused by the prior adoption of a template extinction law in fitting the observed GRB afterglow spectra to derive dust extinction $(\S 2)$. We propose in this work an alternative, robust method based on an analytical formula which can restore the widely adopted template extinction laws $(\S 3)$. For illustration, we apply this approach to GRB 000301C and GRB 021004 ( $\$ 4$ ). We demonstrate in $\S 5$ the uniqueness of the derived extinction laws. The robustness of this approach will be discussed in a separate paper (S. L. Liang \& A. Li 2008a, in preparation) in which the afterglow SEDs of $>50$ GRBs of a wide range of properties are successfully modeled and for which the inferred extinction curves are diverse, with some differing substantially from any of the template extinction curves.

\section{CURRENT STATUS}

At present, the amount of extinction (usually the rest-frame visual extinction $A_{V_{r}}$ ) and the wavelength dependence of the extinction ("extinction curve" or "extinction law"; $A_{\lambda} / A_{V}$ or $A_{\nu} / A_{V}$ if expressed in frequency) are commonly derived by fitting the UV, optical, and near-IR afterglow photometry $\left(F_{\nu}\right.$, with the Galactic extinction corrected) with a power-law model $\left(\propto \nu^{-\beta}\right.$; approximating their intrinsic spectra) reddened by an assumed, template extinction law $A_{\nu} / A_{V}$,

$$
F_{\nu}=F_{o}(\nu / \mathrm{Hz})^{-\beta} \exp \left[-\frac{A_{V_{r}}}{1.086} \frac{A_{(1+z) \nu}}{A_{V_{r}}}\right],
$$

where $\beta$ is the intrinsic power-law slope of the afterglow, $F_{o}$ is a normalization constant (normalized to the overall afterglow flux level), $A_{(1+z) \nu}$ is the rest-frame extinction, and $z$ is the GRB redshift. The factor of 1.086 in equation (1) arises from the conversion of extinction (in magnitude) to optical depth. As a priori, six template extinction laws have been widely adopted in the literature to derive the dust extinction of GRB hosts: (1) a simple power law, $A_{\lambda} / A_{V} \sim \lambda^{-\gamma}$, or even just a linear function of inverse wavelength, $A_{\lambda} / A_{V} \sim \lambda^{-1}$ ("linear" hereafter); (2) the Milky Way (MW) extinction curve (with a prominent bump at $2175 \AA$ ) characterized by $R_{V}$, the total-to-selective extinction ratio (the Galactic average value is $R_{V} \approx 3.1$ ); (3) the featureless Small Magellanic Cloud (SMC) extinction curve, which steeply rises with inverse wavelength from near-IR to far-UV $\left(A_{\lambda} / A_{V} \sim \lambda^{-1.2}\right)$; (4) the Large Magellanic Cloud (LMC) curve, being intermediate between that of the MW and the SMC; (5) the featureless "Calzetti" attenuation law for the dust in local starburst galaxies (Calzetti et al. 1994); ${ }^{6}$ and (6) the relatively flat "Maiolino" extinction law for the dust in the dense circumnuclear region of AGNs (Maiolino et al. 2001), where the dust size distribution is skewed toward large grains (see Fig. 1).

To our knowledge, exceptions to the template extinction approach described here are that of Chen et al. (2006) and Li et al. (2008), both of which were based on the fireball model. The latter approach is limited to bursts of which the X-ray and optical decay indices are the same. In most studies (which assume an extinction template), an SMC-type extinction curve is preferred. This is probably because the $2175 \AA$ extinction feature (which is prominent in the MW and LMC curves) is rarely seen in the

\footnotetext{
${ }^{6}$ We should note that recent Spitzer observations in the near- and mid-IR argue against GRB hosts being strongly starbursting galaxies (Le Floc'h et al. 2006), although their morphological and average radio/submillimeter properties suggest that they are likely massive and actively star-forming galaxies (Berger et al. 2003; Conselice et al. 2005).
}

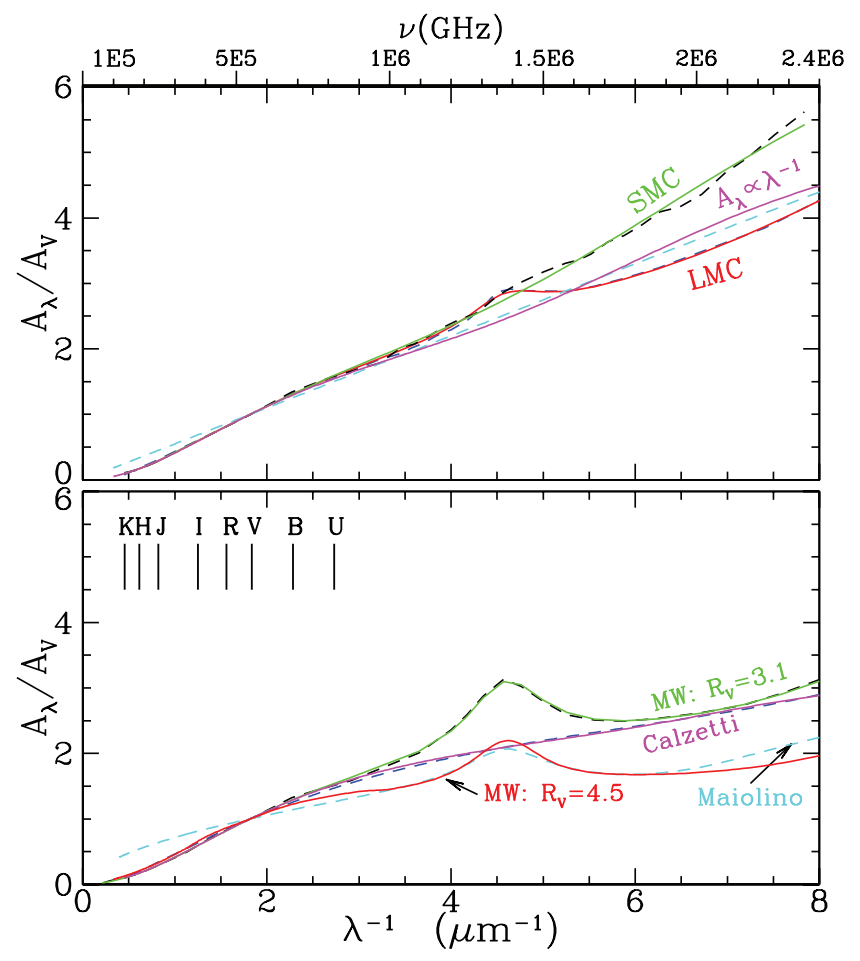

FIG. 1.-Extinction laws widely adopted as "templates" in GRB host extinction studies: the SMC law (top panel, dashed black line; Pei 1992), the LMC law (top panel, dashed blue line; Pei 1992), the linear $A_{\lambda} \propto \lambda^{-1}$ law (top panel, dashed cyan line), the MW Galactic average extinction law $\left(R_{V}=3.1\right.$; bottom panel, dashed black line; Pei 1992), the MW extinction law with $R_{V}=4.5$ for dense clouds (bottom panel, solid red line), the Calzetti starburst attenuation law (bottom panel, dashed blue line), and the Maiolino law for AGN dust tori (bottom panel, dashed cyan line; just like that of the MW with $R_{V}=4.5$ ). Also shown are the "Drude" fits to these "template" extinction laws: SMC (top panel, solid green line), LMC (top panel, solid red line), Linear (top panel, solid magenta line), MW with $R_{V}=3.1$ (bottom panel, solid green line), and Calzetti (bottom panel, solid magenta line).

afterglow spectra of GRBs. So far, its possible detection is only reported in four bursts: GRB 970508 (Stratta et al. 2004), GRB 991216 (Kann et al. 2006; Vreeswijk et al. 2006), GRB 050802 (Schady et al. 2007), and more definitely GRB 070802 (Krühler et al. 2008; Á. Elíasdóttir et al. 2008, in preparation).

However, some studies favor a much flatter or even gray extinction curve (e.g., see Savaglio et al. 2003; Savaglio \& Fall 2004; Stratta et al. 2005; Chen et al. 2006; Li et al. 2008; Perley et al. 2008). With an SMC-type curve, the amount of visual extinction $A_{V}$ or reddening ${ }^{7}$ derived by fitting the afterglow photometry tends to be small since the SMC curve rises so rapidly with $\lambda^{-1}$ that a small $A_{V}$ would imply a large UV extinction. This may explain the finding of "a strong clustering toward low extinction $\left(A_{V} \lesssim\right.$ $0.2 \mathrm{mag}$ )" in a detailed study of 19 GRBs by Kann et al. (2006), and later by Kann et al. (2008) for 15 GRBs. In contrast, for a flatter extinction law like those of Calzetti, Maiolino, MW with $R_{V}>4$, or that derived by Chen et al. (2006), Li et al. (2008), and Perley et al. (2008), a relatively large $A_{V}$ is often obtained.

The visual extinction $A_{V}$ can also be inferred from the dust depletion method based on the gas-phase heavy-element abundances estimated from the afterglow optical absorption spectroscopy

\footnotetext{
${ }^{7}$ Reddening is usually expressed as $E(B-V) \equiv A_{B}-A_{V} \equiv A_{V} / R_{V}$, where $A_{B}$ is the extinction at the $B$ band $\left(\lambda_{B} \approx 4400 \AA\right)$. By definition, gray dust (for which the extinction is just weakly dependent on $\lambda$ ) is characterized by small reddening $E(B-V)$ and large $R_{V}$. Apparently, for gray dust, a small reddening does not necessarily imply a small extinction since $R_{V}$ can be large.
} 
TABLE 1

“'Drude' Fits to Known Extinction Curves for $\lambda=0.1-1 \mu$ m Widely Adopted as "Templates", in Modeling GRB Afterglow SEDs to Derive GRB Host Dust Extinction

\begin{tabular}{|c|c|c|c|c|c|}
\hline Extinction Curve & $c_{1}$ & $c_{2}$ & $c_{3}$ & $c_{4}$ & $\chi^{2 / \text { dof }}$ \\
\hline 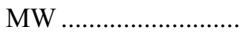 & 14.4 & 6.52 & 2.04 & 0.0519 & 1.66 \\
\hline LMC & 4.47 & 2.39 & -0.988 & 0.0221 & 1.19 \\
\hline SMC $\ldots \ldots \ldots \ldots \ldots \ldots \ldots \ldots \ldots \ldots \ldots \ldots$ & 38.7 & 3.83 & 6.34 & 0 & 1.36 \\
\hline 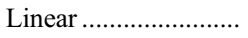 & 66.2 & 4.97 & 22.1 & 0 & 1.42 \\
\hline Calzetti ....................... & 44.9 & 7.56 & 61.2 & 0 & 1.68 \\
\hline
\end{tabular}

(Savaglio et al. 2003; Savaglio \& Fall 2004). This analysis assumes both the dust depletion pattern and the visual extinction per unit dust column $A_{V} / N_{\text {dust }}$ of GRB hosts to be the same as that of the MW. It is quite possible that GRB hosts may have a different depletion pattern and/or a different $A_{V} / N_{\text {dust }}$ conversion factor. The latter could result from a dust composition or size distribution differing from that of the MW.

One can also derive $A_{V}$ from the neutral hydrogen column density $N_{\mathrm{H}}$ derived from Ly $\alpha$ absorption (Hjorth et al. 2003) or the equivalent $N_{\mathrm{H}}$ obtained from soft X-ray absorption (mostly from oxygen K-shell absorption; Galama \& Wijers 2001; Stratta et al. 2004; Watson et al. 2006). There is a puzzling discrepancy between the optical reddening $E(B-V)$ derived from the afterglow SED fitting and the visual extinction $A_{V}$ inferred from the dust depletion analysis or from $N_{\mathrm{H}}$ measured from the Ly $\alpha$ or X-ray absorption spectra, with the former considerably smaller than the latter.

This discrepancy problem could be alleviated if one invokes a flat or gray extinction law. This is because (1) gray dust ( $\gtrsim 1 \mu \mathrm{m})$ characterized with an extinction curve weakly dependent on $\lambda$ in the optical/UV could produce a high $A_{V}$ but little reddening (see footnote 7), and (2) per unit mass gray dust is not as effective as submicron-sized dust in absorbing and scattering optical light so that the $A_{V} / N_{\text {dust }}$ conversion factor for gray dust is smaller than that of the MW dust (with a typical size of $\sim 0.1 \mu \mathrm{m}$; see Li 2008).

The latter would imply that the methods based on dust depletion (see above) and Ly $\alpha / \mathrm{X}$-ray absorption may overestimate $A_{V}$ if the dust size distribution of GRB host galaxies is indeed biased toward large grains, as a result of dust coagulational growth in the dense circumburst environments or preferential destruction of small dust by GRB emission (e.g., see Waxman \& Draine 2000; Fruchter et al. 2001; Perna et al. 2003).

However, we should stress that the gray extinction hypothesis should not be considered as the only solution to the discrepancy problem (after all, SMC-type or even steeper extinction laws were derived for the hosts of some GRBs; see S. L. Liang \& A. Li 2008a, in preparation). Indeed, the mismatch between the X-rayderived $A_{V}$ and that derived from the optical SED modeling could be attributed to physically separate X-ray and optical emission regions (e.g., see Prochaska et al. 2006; Watson et al. 2007). Prochaska et al. (2006) argued that for GRB 051111, the X-ray opacity comes from dust-free gas that is very local to the GRB $(\sim 1 \mathrm{pc})$, while they placed a lower limit of $>50 \mathrm{pc}$ on the host galaxy absorption systems from the GRB. It has also been argued that $A_{V}$ is probably probing the dust outside of the dense molecular cloud around the GRB, since all dust within the cloud is likely to have been obliterated by the burst (e.g., see Perna \& Lazzati 2002; Prochaska et al. 2007). Moreover, if the dust depletion pattern of GRB hosts is different from that of the Milky Way, the discrepancy between the depletion-derived $A_{V}$ and that from the optical SED modeling could be alleviated.

\section{OUR APPROACH}

In view of the shortcomings of the prior assumption of a template extinction law (see $\S 2$ ) and guided by Pei (1992), we propose a simple formula containing four dimensionless parameters $\left(c_{1}, c_{2}, c_{3}\right.$, and $\left.c_{4}\right)$ for the wavelength dependence of the extinction for the dust in GRB host galaxies, instead of adopting any known extinction laws (see $\S 2$ ) as a template:

$$
\begin{aligned}
A_{\lambda} / A_{V} & =\frac{c_{1}}{(\lambda / 0.08)^{c_{2}}+(0.08 / \lambda)^{c_{2}}+c_{3}} \\
& +\frac{233\left[1-c_{1} /\left(6.88^{c_{2}}+0.145^{c_{2}}+c_{3}\right)-c_{4} / 4.60\right]}{(\lambda / 0.046)^{2}+(0.046 / \lambda)^{2}+90} \\
& +\frac{c_{4}}{(\lambda / 0.2175)^{2}+(0.2175 / \lambda)^{2}-1.95}
\end{aligned}
$$

TABLE 2

\begin{tabular}{|c|c|c|c|c|c|c|c|c|c|}
\hline Extinction Type & $c_{1}$ & $c_{2}$ & $c_{3}$ & $c_{4}$ & $\begin{array}{c}A_{V} \\
\text { (mag) }\end{array}$ & $\beta$ & $\begin{array}{c}F_{o} \\
(\mu \mathrm{Jy})\end{array}$ & $\chi^{2} / N_{\text {data }}$ & $\chi^{2} / \mathrm{N}_{\mathrm{dof}}$ \\
\hline \multicolumn{10}{|c|}{ GRB $000301 \mathrm{C}$} \\
\hline Drude & 0.025 & 0.048 & -2.00 & 0 & 0.32 & 0.61 & $3.99 \mathrm{E} 10$ & 0.33 & 1.98 \\
\hline MW & $\ldots$ & $\ldots$ & $\ldots$ & $\ldots$ & 0 & 0.85 & $1.04 \mathrm{E} 14$ & 1.32 & 2.64 \\
\hline SMC & $\ldots$ & $\ldots$ & $\ldots$ & $\ldots$ & 0.11 & 0.62 & 4.68E10 & 0.64 & 1.28 \\
\hline LMC & $\ldots$ & $\ldots$ & $\ldots$ & $\ldots$ & 0 & 0.85 & $1.04 \mathrm{E} 14$ & 1.32 & 2.64 \\
\hline 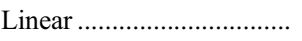 & $\ldots$ & $\ldots$ & $\ldots$ & $\ldots$ & 0.20 & 0.51 & $1.23 \mathrm{E} 9$ & 0.47 & 0.94 \\
\hline 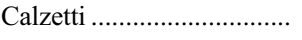 & $\cdots$ & $\ldots$ & $\ldots$ & $\ldots$ & 0 & 0.85 & $1.04 \mathrm{E} 14$ & 1.32 & 2.64 \\
\hline \multicolumn{10}{|c|}{ GRB 021004} \\
\hline Drude..................................... & 0.015 & 0.15 & -2.00 & 0. & 0.13 & 0.78 & 6.13E12 & 0.47 & 1.64 \\
\hline MW & $\ldots$ & $\ldots$ & $\ldots$ & $\ldots$ & 0 & 1.06 & $8.23 \mathrm{E} 16$ & 1.53 & 2.68 \\
\hline 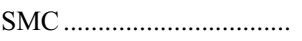 & $\ldots$ & $\ldots$ & $\ldots$ & $\ldots$ & 0.15 & 0.67 & $1.58 \mathrm{E} 11$ & 0.36 & 0.53 \\
\hline LMC ................................... & $\ldots$ & $\ldots$ & $\ldots$ & $\ldots$ & 0 & 1.06 & $8.23 \mathrm{E} 16$ & 1.53 & 2.68 \\
\hline 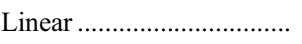 & $\ldots$ & $\ldots$ & $\ldots$ & $\ldots$ & 0.26 & 0.54 & $2.05 \mathrm{E} 9$ & 0.76 & 1.33 \\
\hline 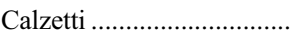 & $\ldots$ & $\ldots$ & $\ldots$ & $\ldots$ & 0.95 & 0 & 46.7 & 0.80 & 1.40 \\
\hline
\end{tabular}

Results of Fitting to the Afterglow SEDs of GRB $000301 \mathrm{C}$ and GRB 021004 with the Drude Approach (see $\S \S 3$ and 4 ) or Various Template Extinction Laws; Note that the Drude Approach has More Free Parameters than the Other Approaches 


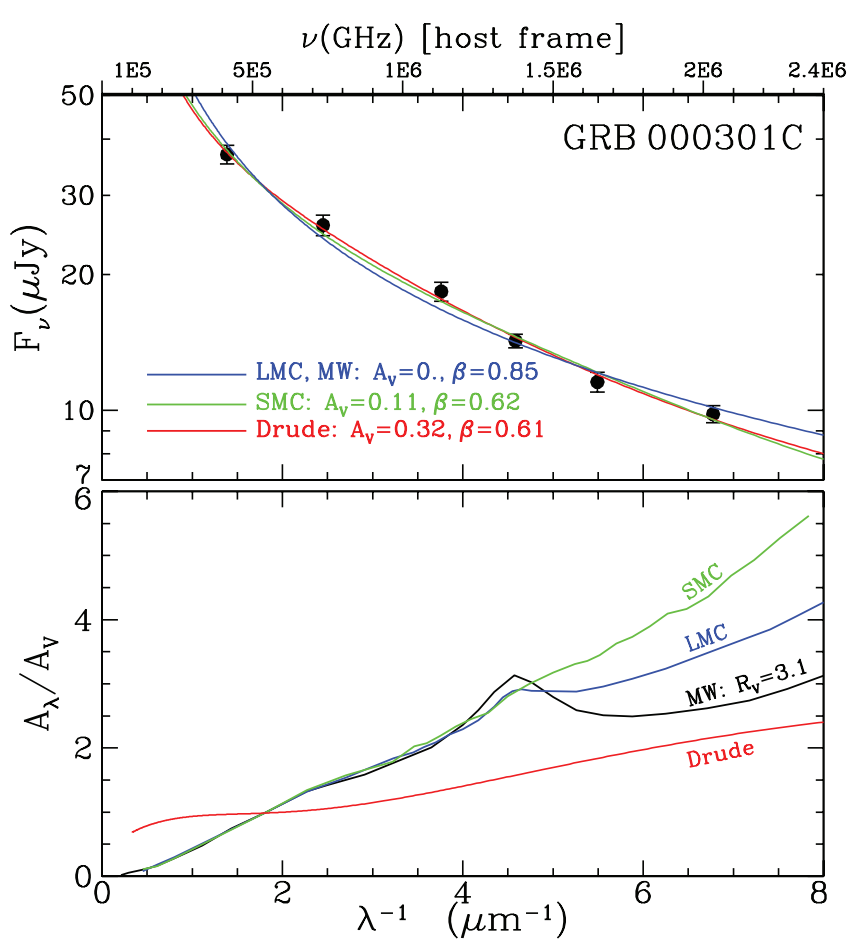

Fig. 2.-Top panel: Fitting the SED of the afterglow of GRB 000301C ( filled black circles) with the SMC (green line) and LMC or MW (blue line) template extinction laws and the "Drude" approach (red; see eq. [2] for the host extinction curve. No extinction is allowed in the MW and LMC models (i.e., the best fit with a MW- or LMC-type extinction is given by $A_{V} \approx 0$ ): a small amount of $A_{V}$ would lead to large deviations from the afterglow SED since the $2175 \AA$ bump prominent in the MW and LMC laws is absent in the afterglow SED. Bottom panel: Comparison of the SMC (green line), LMC (blue line), MW $\left(R_{V}=3.1\right.$; black) extinction laws with that derived from the Drude approach $(r e d)$.

where $\lambda$ is in $\mu \mathrm{m} .{ }^{8}$ While the first term in the right-hand side of equation (2) represents the far-UV extinction rise, the second term and the third term, respectively, account for the near-IR/visible extinction and the $2175 \AA$ extinction bump. We call this the "Drude" approach since equation (2) looks like a sum of Drude functions. As shown in Figure 1, this formula, with the free parameters $c_{j}(j=1, \ldots, 4)$ adjusted using the Levenberg-Marquardt minimization algorithm (Press et al. 1992; see Table 1), can reproduce the extinction curves widely adopted as template extinction laws in GRB afterglow SED modeling, clearly demonstrating the advantages of the proposed formula over any template extinction laws with a fixed wavelength dependence shape: with the widely adopted conventional extinction laws self-contained in equation (2) and the capability of revealing extinction laws differing from the conventional ones, the proposed formula is more flexible and more powerful in modeling the afterglow SEDs. Indeed, as shown in S. L. Liang \& A. Li (2008a, 2008b, in preparation), dust reddening models based on this formula nicely reproduce the observed afterglow SEDs of distant GRBs at $z>4$ (including GRB 050904 at $z \approx 6.3$ ) and that of the "troublesome" GRB

\footnotetext{
${ }^{8}$ Reichart (2001) proposed a seven-parameter formula for the dust extinction curve $A_{\lambda} / A_{V}$ of GRB hosts based on the expressions of Cardelli et al. (1989; "CCM"; for $\lambda>0.3 \mu \mathrm{m}$ ) and of Fitzpatrick \& Massa (1990; "FM"; for $0.1 \mu \mathrm{m}<$ $\lambda<0.3 \mu \mathrm{m}$ ). The problem with the Reichart (2001) formula (see his eqs. [61] and [66]) is that the CCM expression is only valid for the Galactic extinction curves; it is not suitable for the SMC or LMC extinction (Gordon et al. 2003). Therefore, if a GRB host happens to have a SMC- or LMC-type extinction law, models based on the Reichart (2001) formula will not be able to restore the true extinction.
}

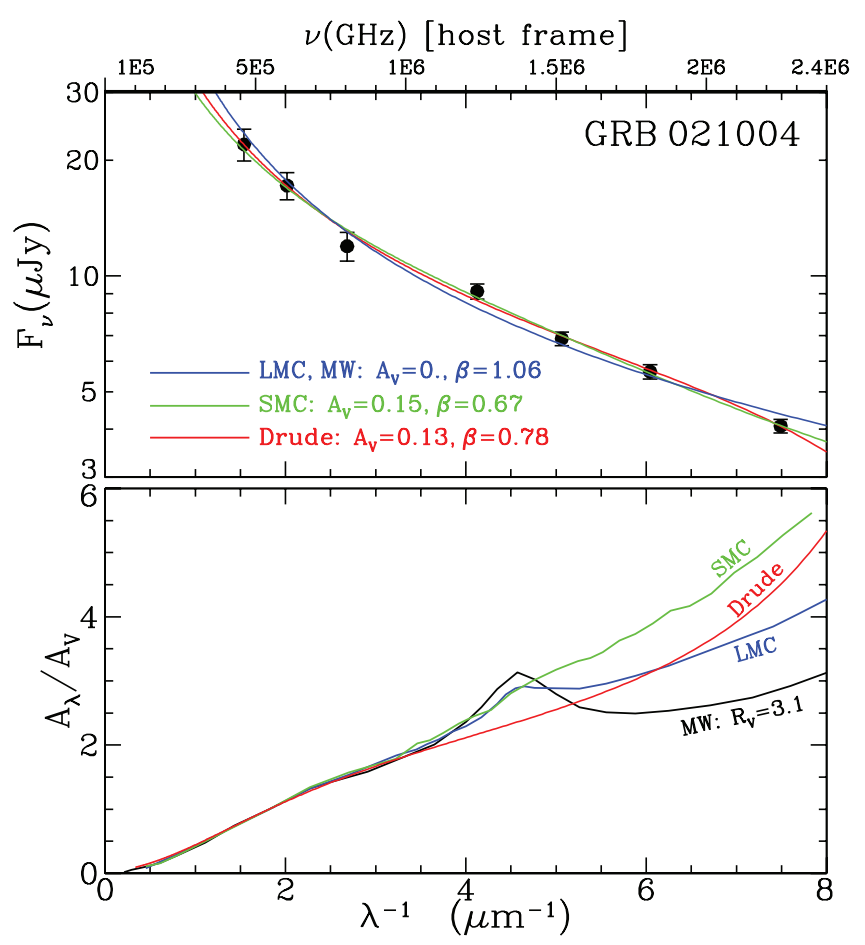

FIG. 3.- Same as Fig. 2, but for GRB 021004.

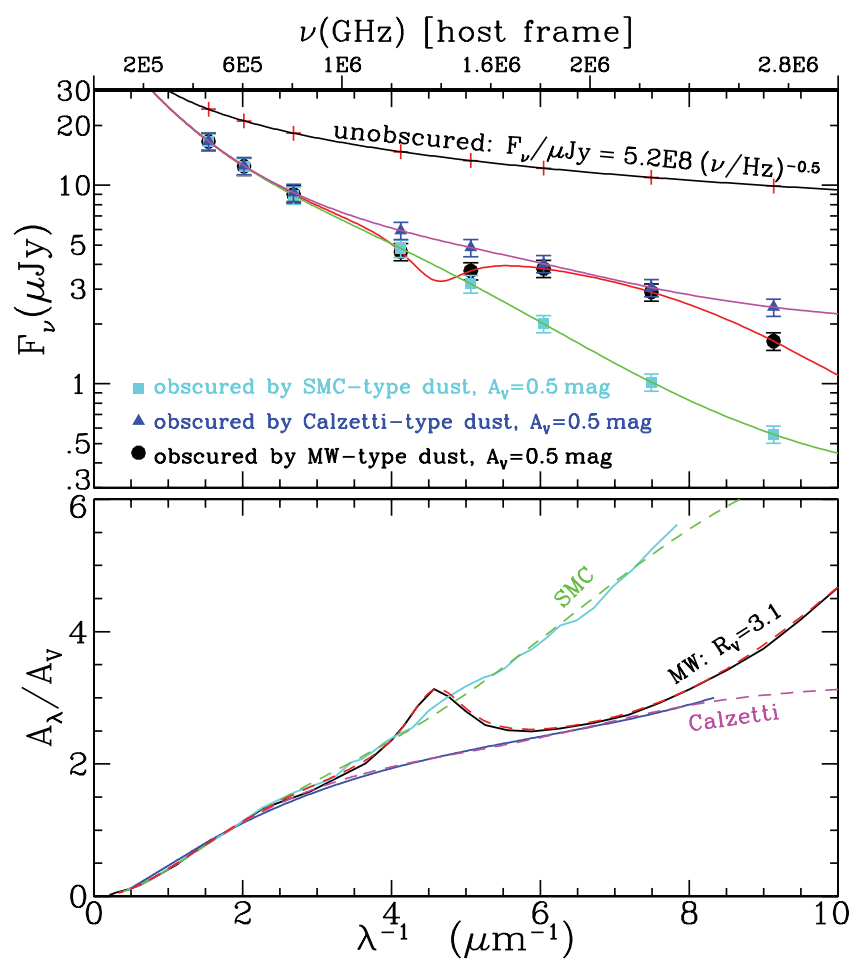

FIG. 4.-Top panel: Drude fits to the observer-frame UBVRIJHK "photometry data" artificially generated by reddening the intrinsic afterglow spectrum $F_{\nu} \propto \nu^{-\beta}$ of a burst at $z \approx 2$ (black line with red crosses superimposed for the observer-frame UBVRIJHK bands) with the SMC ("data," cyan squares; Drude fit, green line), Calzetti ("data," blue triangles; Drude fit, magenta line), and MW ("data," black circles; Drude fit, red line) extinction laws (with $A_{V}=0.5 \mathrm{mag}$ for each). Bottom panel: Comparison of the SMC, Calzetti and MW extinction curves (solid lines) with that inferred from the Drude approach (dashed lines). 
TABLE 3

Results of Drude Fitting to the Artificial Sed Generated by Reddening the Power-Law Afterglow $F_{\nu}(\mu \mathrm{Jy})=5.2 \times 10^{8}(\nu / \mathrm{Hz})^{-0.5}$ with $A_{V}=0.5$ mag Extinction of MW, SMC, and Calzetti-Type (See Fig. 4)

\begin{tabular}{|c|c|c|c|c|c|c|c|c|c|}
\hline Reddening Type & $c_{1}$ & $c_{2}$ & $c_{3}$ & $c_{4}$ & $\begin{array}{c}A_{V} \\
\text { (mag) }\end{array}$ & $\beta$ & $\begin{array}{c}F_{o} \\
(\mu \mathrm{Jy})\end{array}$ & $\chi^{2} / N_{\text {data }}$ & $\chi^{2} / N_{\text {dof }}$ \\
\hline MW & 14.3 & 6.49 & 2.02 & 0.0514 & 0.501 & 0.499 & $5.24 \mathrm{E} 8$ & $3.26 \mathrm{E}-4$ & $4.35 \mathrm{E}-4$ \\
\hline SMC ……………............ & 39.4 & 3.89 & 6.31 & 0. & 0.500 & 0.501 & $5.26 \mathrm{E} 8$ & $1.32 \mathrm{E}-3$ & $1.76 \mathrm{E}-3$ \\
\hline Calzetti .............................. & 45.2 & 7.51 & 61.7 & 0. & 0.497 & 0.502 & $5.17 \mathrm{E} 8$ & $7.98 \mathrm{E}-4$ & $1.06 \mathrm{E}-3$ \\
\hline
\end{tabular}

061126 (Perley et al. 2008) without resorting to an exotic extinction law.

\section{GRB 000301C AND GRB 021004: TEST CASES}

We apply the above-described technique ( $\S 3$ ) to the optical afterglows of GRB $000301 \mathrm{C}$ at $z \approx 2.04$ (Jensen et al. 2001) and GRB 021004 at $z \approx 2.33$ (Fynbo et al. 2005). They are selected mainly because they are among the best-observed in terms of sampling in the time domain and multiwavelength coverage. We fit their broadband SEDs using equations (1) and (2) with $\beta, A_{V}$, $c_{1}, c_{2}$, and $c_{3}$ allowed to vary as free parameters $\left[F_{o}\right.$ is not really a free parameter; for a given set of $\left(\beta, A_{V}, c_{1}, c_{2}, c_{3}\right), F_{o}$ is uniquely determined by the overall flux level. Therefore, in the SED modeling we fit five free parameters to the six (seven) data points of GRB 000301C (GRB 021004)]. ${ }^{9}$ We derive the best-fit parameters based on the Levenberg-Marquardt minimization algorithm (see Table 2). As shown in Figure 2 for GRB 000301C and in Figure 3 for GRB 021004, almost perfect fits to the observed SEDs are achieved through this approach. The inferred extinction curves differ substantially from any of the template extinction laws.

\section{DISCUSSION}

We have also fitted the afterglow SEDs of GRB $000301 \mathrm{C}$ and GRB 021004 in terms of the MW, SMC, LMC, Calzetti, and "linear" template extinction curves (see Table 2 and Figs. 2 and 3). Since for a given template extinction law the wavelength dependence of the extinction $A_{\lambda} / A_{V}$ is fixed, we are now left with only three parameters: $F_{o}, \beta$, and $A_{V}$.

The models based on the MW and LMC extinction laws could not fit the observed SEDs at all. This is because the $2175 \AA$ extinction feature which is prominent in the MW and LMC curves is absent in the SEDs of GRB 000301C and GRB 021004. In contrast, the SMC and "linear" models closely fit the afterglow SEDs of these two bursts, better than the Drude model proposed here as measured by $\chi^{2} / N_{\text {dof }}$ (see Table 2 ).

While the Drude model has three more parameters than the SMC and "linear" models, the quality of the fitting of the Drude model is even not as good as that of the SMC or "linear" model. Then, why do not we simply adopt the SMC or "linear" model?

First of all, we should note that there are no physical reasons for a prior assumption of a known extinction law, either that of the SMC, LMC, "linear" or MW: the composition and size distribution (and therefore the extinction law) of the dust in the dense circumburst clouds of GRB hosts with a wide range of metallicities and evolutionary stages are not expected to resemble that of the MW, LMC, or SMC (e.g., see Dwek 2005). In the literature, a

\footnotetext{
${ }^{9}$ We set $c_{4}=0$ based on a visual inspection of the observed SEDs which clearly suggest the absence of a $2175 \AA$ A feature (see Figs. 2 and 3). With $c_{4}$ treated as a free, positive parameter, even the best fits (given by $c_{4} \approx 0.0034$ and 0.0018 for GRB 000301C and GRB 021004, respectively; for comparison, $c_{4} \approx 0.051$ and 0.039 for MW and LMC, respectively) are not as good as that provided by models with $c_{4}=0$. We place an upper limit of $c_{4} \approx 0.015(0.0073)$ for GRB 000301C (GRB 021004).
}

SMC-type extinction is often assumed for low-metallicity environments. However, there is no physical basis for this (except the lack of grain growth in these regions because of the lack of raw dust materials - the SMC dust, on average, is substantially smaller than that of the Milky Way; see Weingartner \& Draine 2001). Moreover, it is known that the GRB hosts have a wide range of metallicities. Indeed, the reasons why the MW, LMC, and SMC laws are often used for GRB afterglow SED modeling are mainly (1) that little is known about the extinction laws of other galaxies, and (2) the Pei (1992) formula for the MW, LMC, and SMC extinction laws is numerically convenient for computer implementation.

Second, although the SMC-type extinction is preferred in most of the present afterglow SED modeling studies, only the Drude approach is capable of reproducing the SEDs of those reddened by gray extinction or by nonconventional extinction. Indeed, it was shown that the afterglow SED of GRB 050904 at a redshift of $z \approx 6.3$ cannot be explained by dust reddening with any of the conventional (MW, SMC, Calzetti) extinction curves; instead, it can be well reproduced by invoking the extinction curve inferred for a distant quasar at $z=6.2$ (Maiolino et al. 2004), suggesting that the properties of dust may evolve beyond $z=6$ (Stratta et al. 2007).

Third, the Drude model would at least complement the models using template extinction curves, particularly for those bursts for which the Drude model gives a larger $\chi^{2} / N_{\text {dof }}$ (but still fits the observed SEDs well). Given that the derived extinction $A_{V}$ and the intrinsic spectral slope $\beta$ differ appreciably among different approaches (see Table 2), the SMC model (and other models) should be used along side with the Drude model to gain insight into the "true" extinction and the "true" spectral slope.

We finally demonstrate the uniqueness of the extinction curve inferred from the Drude approach. To this end, we generate three sets of afterglow "photometry data" by reddening the intrinsic afterglow spectrum $F_{\nu}(\mu \mathrm{Jy})=5.2 \times 10^{8}(\nu / \mathrm{Hz})^{-0.5}$ of a burst at $z \approx 2$, respectively, with three template extinction laws: MW, $\mathrm{SMC}$, and Calzetti, each with $A_{V}=0.5 \mathrm{mag}$. We then apply the Drude approach to these three sets of artificially created GRB afterglow data. As shown in Figure 4, we uniquely restore the MW, SMC, and Calzetti extinction laws: the inferred extinction curves are almost identical to that used to redden the intrinsic spectrum (the derived parameters [see Table 3] are essentially the same as those tabulated in Table 1).

We thank the anonymous referee and J. X. Prochaska for very helpful comments. A. L. and S. L. are supported in part by a NASA/ Swift Theory Program, a NASA/Chandra Theory Program, and the NSFC Outstanding Oversea Young Scholarship. D. M. W. is supported by the NSFC grants 10621303 and 10673034, and the National Basic Research Program of China (973 Program 2007CB815404). D.A.K. and S. K. acknowledge financial support by DFG grant K1 766/13-2. 
REFERENCES

Berger, E., Cowie, L. L., Kulkarni, S. R., Frail, D. A., Aussel, H., \& Barger, A. J. 2003, ApJ, 588, 99

Calzetti, D., Kinney, A. L., \& Storchi-Bergmann, T. 1994, ApJ, 429, 582

Cardelli, J. A., Clayton, G. C., \& Mathis, J. S. 1989, ApJ, 345, 245

Chen, S. L., Li, A., \& Wei, D. M. 2006, ApJ, 647, L13

Conselice, C. J., et al. 2005, ApJ, 633, 29

Djorgovski, S. G., Frail, D. A., Kulkarni, S. R., Bloom, J. S., Odewahn, S. C., \& Diercks, A. 2001, ApJ, 562, 654

Djorgovski, S. G., Kulkarni, S. R., Bloom, J. S., Goodrich, R., Frail, D. A., Piro, L., \& Palazzi, E. 1998, ApJ, 508, L17

Dwek, E. 2005, in AIP Conf. Proc. 761, The Spectral Energy Distributions of Gas-Rich Galaxies: Confronting Models with Data, ed. C. C. Popescu \& R. J. Tuffs (Melville: AIP), 103

Fiore, F., Guetta, D., Piranomonte, S., D’Elia, V., \& Antonelli, L. A. 2007, A\&A, 470,515

Fitzpatrick, E. L., \& Massa, D. L. 1990, ApJS, 72, 163

Fruchter, A., Krolik, J. H., \& Rhoads, J. E. 2001, ApJ, 563, 597

Fynbo, J. P. U., et al. 2005, ApJ, 633, 317

Galama, T. J., \& Wijers, R. A. M. J. 2001, ApJ, 549, L209

Gordon, K. D., Clayton, G. C., Misselt, K. A., Landolt, A. U., \& Wolff, M. J. 2003, ApJ, 594, 279

Hjorth, J., et al. 2003, ApJ, 597, 699

Jakobsson, P., et al. 2004, A\&A, 427, 785

Jaunsen, A. O., et al. 2008, ApJ, 681, 453

Jensen, B. L., et al. 2001, A\&A, 370, 909

Kann, D. A., Klose, S., \& Zeh, A. 2006, ApJ, 641, 993

Kann, D. A., et al. 2008, ApJ, submitted (arXiv: 0712.2186)

Klose, S., et al. 2000, ApJ, 545, 271

Krühler, T., et al. 2008, ApJ, 685, 376

Le Floc'h, E., Charmandaris, V., Forrest, W. J., Mirabel, I. F., Armus, L., \& Devost, D. 2006, ApJ, 642, 636

Levan, A., et al. 2006, ApJ, 647, 471

Li, A. 2008, in Small Bodies in Planetary Sciences, ed. I. Mann, A. Nakamura, \& T. Mukai (Berlin: Springer), 167
Li, Y., Li, A., \& Wei, D. M. 2008, ApJ, 678, 1136

Maiolino, R., Marconi, A., \& Oliva, E. 2001, A\&A, 365, 37

Maiolino, R., Schneider, R., Oliva, E., Bianchi, S., Ferrara, A., Mannucci, F., Pedani, M., \& Roca Sogorb, M. 2004, Nature, 431, 533

Osterbrock, D. E., \& Ferland, G. J. 2006, Astrophysics of Gaseous Nebulae and Active Galactic Nuclei (2nd ed; Sausalito: Univ. Science Books)

Pei, Y. C. 1992, ApJ, 395, 130

Perley, D. A., et al. 2008, ApJ, 672, 449

Perna, R., \& Lazzati, D. 2002, ApJ, 580, 261

Perna, R., Lazzati, D., \& Fiore, F. 2003, ApJ, 585, 775

Press, W. H., Teukolsky, S. A., Vetterling, W. T., \& Flannery, B. P. 1992, Numerical Recipes in FORTRAN: The Art of Scientific Computing (2nd ed.; Cambridge: Cambridge Univ. Press)

Prochaska, J. X., Chen, H.-W., \& Bloom, J. S. 2006, ApJ, 648, 95

Prochaska, J. X., Chen, H.-W., Dessauges-Zavadsky, M., \& Bloom, J. S. 2007, ApJ, 666, 267

Ramirez-Ruiz, E., Trentham, N., \& Blain, A. W. 2002, MNRAS, 329, 465

Reichart, D. E. 2001, ApJ, 553, 235

Savaglio, S., \& Fall, S. M. 2004, ApJ, 614, 293

Savaglio, S., Fall, S. M., \& Fiore, F. 2003, ApJ, 585, 638

Schady, P., et al. 2007, MNRAS, 377, 273

Stratta, G., Fiore, F., Antonelli, L. A., Piro, L., \& De Pasquale, M. 2004, ApJ, 608,846

Stratta, G., Perna, R., Lazzati, D., Fiore, F., Antonelli, L. A., \& Conciatore, M. L. 2005, A\&A, 441, 83

Stratta, G., Maiolino, R., Fiore, F., \& D’Elia, V. 2007, ApJ, 661, L9

Tanvir, N. R., et al. 2008, MNRAS, 388, 1743

Vreeswijk, P. M., et al. 2006, A\&A, 447, 145

Watson, D., et al. 2006, ApJ, 652, 1011

Watson, D., Hjorth, J., Fynbo, J. P. U., Jakobsson, P., Foley, S., Sollerman, J., \& Wijers, R. A. M. J. 2007, ApJ, 660, L101

Waxman, E., \& Draine, B. T. 2000, ApJ, 537, 796

Weingartner, J. C., \& Draine, B. T. 2001, ApJ, 548, 296

Woosley, S. E., \& Bloom, J. S. 2006, ARA\&A, 44, 507 